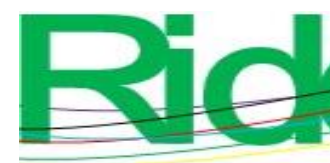

Artículos científicos

\title{
La disparidad del sistema escolarizado y semiescolarizado: estudio de egresados de la carrera de Abogado
}

\author{
The disparity of the schooled and semi-schooled system: A Law School \\ graduates study

\begin{abstract}
A disparidade do sistema escolar e do semi-escolar: estudo dos licenciados em Direito
\end{abstract} \\ Ana Gabriela González Anaya \\ Universidad de Guadalajara, Centro Universitario de los Altos, México \\ ana.ganaya@academicos.udg.mx \\ https://orcid.org/0000-0003-4075-1768
}

\section{Resumen}

La elección de una carrera suele basarse en el interés existente por esta, pero en ocasiones la decisión es influenciada por otros factores, tales como la cercanía al campus, las posibilidades económicas del futuro universitario e incluso el horario disponible. En este trabajo se presenta el análisis de dos grupos de egresados de la misma licenciatura (carrera de Abogado), pero en dos modalidades distintas: la escolarizada y la semiescolarizada. Ambos grupos fueron consultados mediante una entrevista semiestructurada. Los resultados fueron dispares en cuanto a la edad, los días que asistían a clases y la forma de sustentar sus estudios. En este trabajo se expone que la modalidad de estudio les llevó a tener experiencias opuestas, por ejemplo, en cuanto al apoyo que recibieron por parte del campus. En síntesis, en ambos grupos se analiza cómo el capital humano puede ser o no una influencia para obtener un mejor empleo y posicionamiento en el mundo laboral.

Palabras clave: abogado, educación alternativa, enseñanza, estudiante, graduado. 


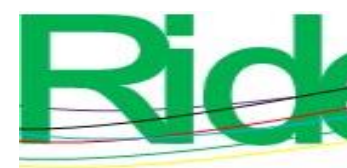

Revista Iberoamericana para la Investigación y el Desarrollo Educativo ISSN $2007-7467$

\section{Abstract}

The choice of a career is usually based on the existing interest in it, but sometimes the decision is influenced by other factors such as the proximity of the campus, the economic possibilities of the future university and also the schedule. This work presents the analysis of two groups of graduates of the same degree: Lawyer's Degree, but in two different modalities, the schooled and the semi-schooled. Both groups were contacted and the results of the semiestructured interview that was applied are shown here.

Both groups have differences, among them we find their ages, their schedules and how they supported themselves through their university studies. In this study it can be shown that the chosen modality made them go through diametrically different experiences with respect to the students of the school system: the support they received from the campus was perceived as lees, although not necessarily so, and they stated that they had gone through this university journey in a hastier way. Both groups analyze how human capital may or may not be influencing to obtain a better job and position in the labor market.

Keywords: lawyer, alternative education, teaching, student, graduate.

\section{Resumo}

A escolha de uma carreira geralmente é baseada no interesse existente na mesma, mas às vezes a decisão é influenciada por outros fatores, como a proximidade do campus, as possibilidades econômicas da futura universidade e até os horários disponíveis. Este artigo apresenta a análise de dois grupos de concluintes da mesma licenciatura (licenciatura em Direito), mas em duas modalidades distintas: os escolarizados e os semi-escolarizados. Ambos os grupos foram consultados por meio de entrevista semiestruturada. Os resultados foram desiguais em termos de idade, dias de aulas e modo de apoiar os estudos. Neste trabalho afirma-se que a modalidade de estudo os levou a vivenciar experiências opostas, por exemplo, no que diz respeito ao apoio que recebiam do campus. Em suma, ambos os grupos analisam como o capital humano pode ou não ser uma influência para obter um melhor emprego e posição no mundo do trabalho.

Palavras-chave: advogado, educação alternativa, ensino, estudante, pós-graduação.

Fecha Recepción: Julio 2020

Fecha Aceptación: Diciembre 2020 


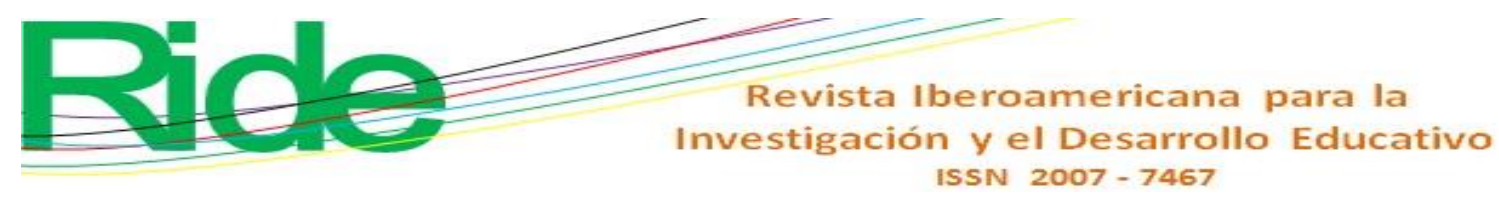

\section{Introducción}

El seguimiento de egresados es un trabajo con el que se pretende describir los resultados de la formación educativa de un grupo — en este caso— de la carrera de Abogado ${ }^{1}$, así como la manera en que se han insertado en el ámbito laboral. Esto con el objetivo de obtener información sobre el entorno laboral de los profesionistas, las dificultades que han encontrado en el campo de su profesión y la manera en que su formación académica ha influido para facilitar o dificultar el acceso al mundo del trabajo. Por ello, la información recabada con este tipo de estudios permite a las instituciones educativas conocer sus debilidades, fortalezas y áreas de trabajo que deben optimizarse.

Esta indagación es distinta a otros seguimientos de egresados, ya que se basa en las respuestas de un grupo de profesionistas cuyo paso por la universidad fue a través de dos sistemas: el escolarizado y el semiescolarizado. Este último se caracteriza porque el alumno asiste a clases un solo día a la semana y porque el énfasis del trabajo asignado recae en las actividades y proyectos que el estudiante realiza por cuenta propia.

Otra particularidad que diferencia a ambos grupos se encuentra en que algunos de los alumnos entrevistados pertenecientes al sistema semiescolarizado ya tenían cursadas licenciaturas previas. Esto significa que la carrera de Abogado constituye un complemento o una manera de seguir activos en el mundo académico debido a que ya se habían jubilado de sus empleos anteriores.

Hecha esta diferenciación, se puede decir que el presente trabajo ofrece resultados comparativos entre dos sistemas escolares que confluyen en un campus y que comparten un mismo programa de estudios. Se presentan, por ende, similitudes y diferencias entre aquellos que - aunque estudiaron la misma carrera - tuvieron condiciones distintas tanto en el aspecto académico como en sus objetivos profesionales.

Los hallazgos se presentan divididos en dos partes: por un lado, se detallan las características de los estudiantes durante su formación profesional, origen social y expectativas que tenían con respecto a su carrera; por otra parte, se describen las características de las trayectorias profesionales de los egresados una vez que dejaron la institución. Asimismo, se presentan gráficas y resultados de ambos sistemas para facilitar la comparación. Cabe señalar que al ser un trabajo comparativo se encontró que algunos

\footnotetext{
${ }^{1}$ En el caso de la Universidad de Guadalajara, se le conoce como carrera de Abogado, y no licenciatura en Derecho. Decidí mantener el término utilizado por dicha universidad.
} 


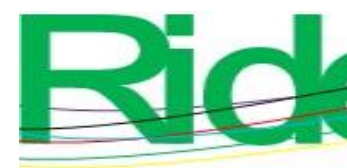

Revista Iberoamericana para la Investigación y el Desarrollo Educativo ISSN 2007 - 7467

egresados contaban con una trayectoria laboral de varios años o incluso décadas, de ahí que algunos resultados sean distintos a aquellos donde los abogados se encontraban buscando sus primeros empleos o acercamientos al mercado laboral.

De acuerdo con las cifras de la Encuesta Nacional de Ocupación y Empleo (2015), en 2012 (momento de egreso de los sujetos encuestados), en Jalisco 44448 personas se dedicaban al ejercicio del derecho en su trabajo, cifra que aumentó a 52712 en el año 2015. Se presentan datos de esa fecha, ya que es en la que egresaron los sujetos de estudio para esta investigación. En el estado de Jalisco, en dicho periodo los abogados tenían un salario mensual promedio de $\$ 9506$ pesos mexicanos (el promedio de ingresos mensuales de los profesionistas era de $\$ 10600$ pesos). En otras palabras, los egresados de la referida carrera tenían un salario que se hallaba por debajo del promedio.

Por otra parte, $43.9 \%$ de quienes son profesionistas y trabajan en el estado de Jalisco son mujeres, mientras que el porcentaje de la carrera de Abogado (o licenciatura en Derecho) es de $39.4 \%$. Las principales ocupaciones de los egresados de dicha licenciatura son abogado y asesor jurídico, analista y proyectista jurídico, investigador jurídico y defensor de oficio (Instituto Nacional de Estadística y Geografía [Inegi], 2015). A continuación, se analiza cómo es la disparidad entre los grupos escolares seleccionados.

\section{Teoría del capital humano}

La teoría del capital humano se toma como base para este trabajo. Se pretende demostrar que los sujetos invirtieron en su propio capital al formarse en una licenciatura (en este caso, Derecho o la denominada carrera de Abogado en el Centro Universitario de los Altos, de la Universidad de Guadalajara, México). La adquisición de nuevas habilidades, conocimientos y desarrollo de sus aptitudes llevaría a los egresados a tener mayores y mejores oportunidades cuando se empiezan a desenvolver en el mercado laboral.

La propuesta de Schultz (Schultz 1983; González y Ortega, 2015; Villalobos y Pedroza, 2009) explicaría la pretensión de los individuos de invertir en su propia educación. Vale destacar que en el presente trabajo los egresados tuvieron diferentes formas de invertir en su educación, ya que mientras unos podían hacerlo asistiendo a clases presenciales durante toda la semana, otros elegían solo los fines de semana, por lo que dependían de sus propios avances. Estos últimos, como ya se mencionó, contaban con un trabajo o con una licenciatura 


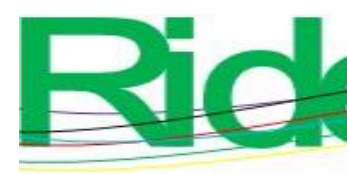

Revista Iberoamericana para la Investigación y el Desarrollo Educativo

ISSN 2007 - 7467

previa, de ahí que las razones por las que invertían en su propio capital eran distintas a las del otro grupo analizado.

Hay estudios que relacionan el crecimiento económico de una nación con el papel que tiene la educación y, por lo tanto, con el capital humano (Villalobos y Pedroza, 2009). Por ello, se podría entender como un punto de atracción de inversión en capital físico, aunque el capital humano también se puede asociar con un mejor desarrollo y posterior éxito del uso de nuevas tecnologías. En otras palabras, más años de escolaridad de un sujeto se podrían asociar con la aspiración a un mejor empleo, lo que se podría reflejar en el crecimiento económico de la región y del país (Guerrero, 2020).

Algunas características propias del capital humano son las siguientes: no puede transferirse a otro sujeto, ya que solo lo posee quien ha hecho la inversión; existe mientras el sujeto lo esté empleando; suele adquirirse en la juventud (punto que contrasta con el de los egresados que comenzaron sus estudios a los 40 o 50 años de edad), y no se devalúa (Villalobos y Pedroza, 2009).

El caso mexicano, visto desde la economía, muestra un lento crecimiento económico. Una de las explicaciones se halla en las restricciones de capital humano, ya que no se está contratando a los trabajadores con mayores calificaciones y niveles educativos, a pesar de que la cobertura educativa y la escolaridad de población han aumentado (Székely y Flores, 2018). Si a esto se suma que hay un gran número de sujetos interesados en invertir en sí mismos, pero que las condiciones en las que se encuentran los obligan a recurrir a fuentes de educación no tradicionales (p. ej., sistema semiescolarizado o educación en línea), es posible entrever que la calidad de la educación será distinta. Esto, lógicamente, no se debe a que las demás opciones sean deficientes, sino que no se les ofrece las mismas oportunidades que a otros estudiantes, como en el caso de los egresados del sistema semiescolarizado, los cuales consideran que no tuvieron las mismas facilidades que los estudiantes del sistema escolarizado para acceder a los libros de la biblioteca, al uso del equipo de cómputo o al laboratorio de idiomas, lo cual les limita en sus aspiraciones en el mercado laboral (Guerrero, 2020; Organización para la Cooperación y el Desarrollo Económico [OCDE], 2010).

Además de conocer si la inversión que estos egresados hicieron en su propio capital les ha sido redituable o significativa (traducida en mejores puestos de trabajo, ascensos, posibilidades laborales, actividades relacionadas con su profesión), resulta significativo conocer cómo los sujetos de ambos grupos (cuyos padres en la mayoría de los casos no 


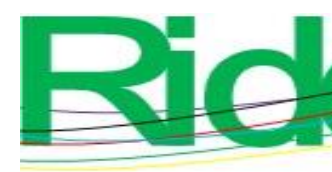

Revista Iberoamericana para la Investigación y el Desarrollo Educativo ISSN 2007 - 7467

contaban con estudios universitarios) superaron los obstáculos académicos que se presentaban.

Por otra parte, la apuesta por una mejor educación, vista como un derecho e implementada en México con mayor ahínco a partir de los años noventa, ha traído resultados cuyo impacto podemos observar en un aumento en el ingreso per cápita y en un mejor acceso a la salud (Jiménez y Pacheco, 2018).

\section{Metodología}

Ambos grupos de estudiantes fueron admitidos en el calendario escolar 2008 en el Centro Universitario de los Altos. Se eligió trabajar con esta cohorte estudiantil por dos razones: 1) su egreso fue en 2012, por lo que al momento del análisis ya se habían desempeñado en el mercado laboral (esto permitió determinar la influencia de haber cursado una carrera universitaria para conseguir un empleo); 2) fue la última cohorte generacional de la carrera de Abogado de quienes se recopiló información como egresados, lo que sirvió para comparar a ambos grupos.

Se trabajó con una metodología cualitativa. En algunos casos, se realizaron entrevistas personales con preguntas abiertas y cerradas, mientras que en otros solo se efectuaron llamadas telefónicas, ya que esta fue la manera elegida por los sujetos de estudio. El instrumento fue diseñado por profesores del campus al que pertenece la carrera analizada; a este se denominó Cuestionario de seguimiento de egresados de Cualtos. Debido a que se emplearon entrevistas semiestructuradas, el cuestionario funcionó como una guía para las preguntas formuladas; si en una respuesta se incluía lo correspondiente a dos o más preguntas, se omitía la mención de estas. Se buscó a la totalidad de egresados titulados de ambos grupos y se consideró a aquellos que accedieron a responder todo el cuestionario.

En el caso de los estudiantes de la modalidad escolarizada de la carrera de Abogado, $62 \%$ de quienes ingresaron continuaron hasta culminar con sus estudios, mientras que en la modalidad semiescolarizada la eficiencia terminal fue de $43.47 \%$, la más baja de las 14 opciones profesionales que ofrece el campus, aunque debemos tomar en cuenta que a nivel nacional la relación entre alumnos inscritos y los que finalizan sus estudios es de $32.20 \%$ (González y Villanueva, 2007). Esto indica que aunque los índices de eficiencia terminal de ambos grupos se encuentran muy por debajo de otros pertenecientes al mismo centro 

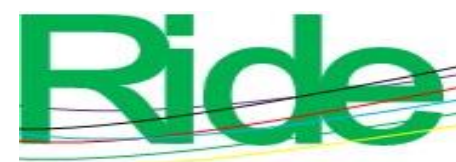

Revista Iberoamericana para la Investigación y el Desarrollo Educativo

ISSN 2007 - 7467

universitario, los dos porcentajes son mayores al promedio en las universidades de todo México.

Para la obtención del porcentaje de eficiencia terminal se tomó en cuenta el número de alumnos inscritos en una generación (sin incluir a aquellos estudiantes rezagados o que reprobaron asignaturas y pasaron a formar parte de este grupo a pesar de haberse inscrito anteriormente). Se les conoce también como generaciones "sin contaminación”, ya que se toma en cuenta solo a aquellos que fueron aceptados como parte de ese grupo inicialmente.

El universo de estudio se conformó por el total de egresados; sin embargo, aunque las dificultades para localizarlos fueron similares a las que ocurren con otros trabajos de este tipo (Jaramillo, Pineda y Ortiz Correa, 2006), se logró entablar comunicación con la mayoría de ellos. En este trabajo se ofrecen los resultados de los egresados encuestados $(28 \%$ de semiescolarizado y $58 \%$ de escolarizado) expresados como el $100 \%$ de cada grupo para facilitar la comprensión de los resultados. Es importante señalar que en el caso de aquellos que estudiaron de forma semiescolarizada la negativa a participar fue como respuesta al trato que dijeron haber recibido de la universidad como alumnos. Las dificultades que tuvieron a lo largo de sus estudios universitarios tuvieron relación con la modalidad que estaban atendiendo, y no tanto con el programa de estudios, como se podrá observar más adelante.

Figura 1. Eficiencia terminal

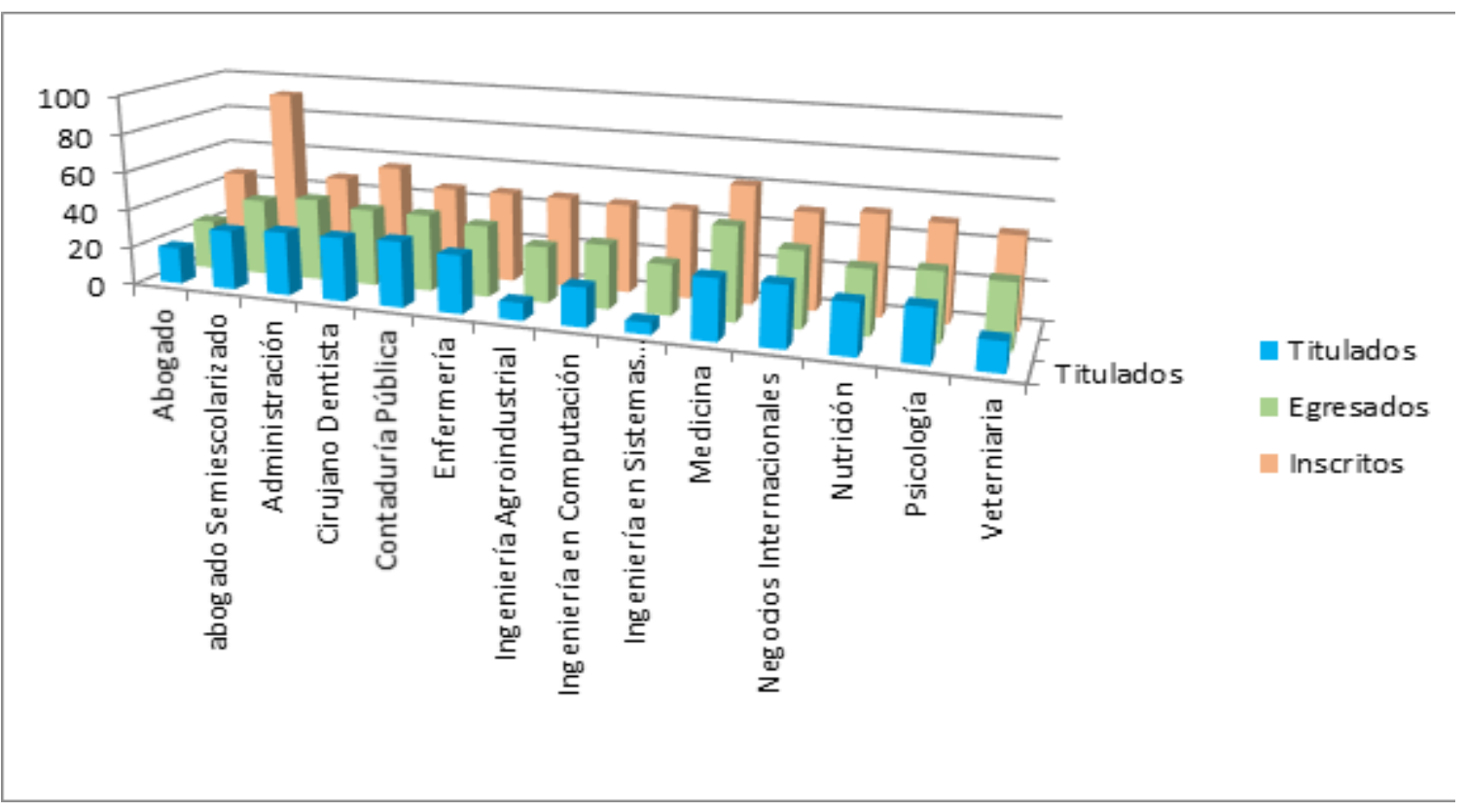

Fuente: González y Ortega (2015) 


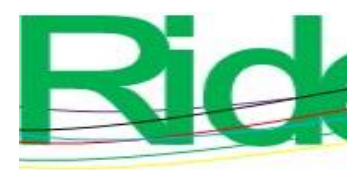

Revista Iberoamericana para la Investigación y el Desarrollo Educativo

ISSN 2007 - 7467

Además de preguntar a los egresados sobre su desarrollo como estudiantes y sobre su posterior desenvolvimiento en el ámbito laboral, se buscó recabar información acerca de la formación de sus padres, las problemáticas enfrentadas a lo largo de sus estudios, las recomendaciones que harían, así como sus propias percepciones de los conocimientos adquiridos. Para ello, los egresados fueron invitados a expresarse libremente.

En los resultados de este estudio las edades de los egresados fluctuaron entre los $24 \mathrm{y}$ los 65 años de edad. Esta es una de las grandes diferencias entre los alumnos del sistema escolarizado y los del semiescolarizado, pues los primeros suelen hallarse en un rango de edad de entre los 18 a los 22 años cuando ingresan a la universidad.

De los egresados del sistema semiescolarizado, $46.6 \%$ son hombres y $53.3 \%$ mujeres, mientras que en el escolarizado $38.8 \%$ son varones y $61.1 \%$ mujeres. En cuanto al estado civil, $40 \%$ de los egresados de semiescolarizado están casados, $6.6 \%$ vive en unión libre y $40 \%$ son solteros (cabe señalar que no todos los entrevistados respondieron a esta pregunta). Entre los egresados de la carrera en modalidad escolarizada, $22.2 \%$ son casados y $72.2 \%$, solteros. Se presenta a continuación el análisis de resultados.

\section{Resultados}

En su gran mayoría los abogados habían elegido esta carrera como una primera opción: 60 \% de los egresados de semiescolarizado así lo manifestaron, mientras que $83.3 \%$ de la otra modalidad coincidieron con esa idea. En el caso de los egresados de semiescolarizado, $20 \%$ contaba ya con otra licenciatura antes de ingresar a la carrera de Abogado. Entre las otras carreras solicitadas, Contaduría fue la elegida como primera opción.

En cuanto a las razones por las que decidieron convertirse en abogados, los egresados de semiescolarizado manifestaron lo siguiente: $40 \%$ porque la oferta de clases era una sola vez por semana, $33.3 \%$ por el interés que despertó en ellos la profesión, $13.3 \%$ porque próximamente se jubilarían (esta última justificación no se relaciona con la vinculación de esta carrera y la labor desempeñada; de hecho, es probable que tampoco fueran a ejercer la abogacía), y $6.6 \%$ porque la carrera era un complemento para la que ya tenían.

En el caso de los egresados de escolarizado, $72.2 \%$ expresó que eligieron la carrera por el interés que sentían. Otras razones con menores porcentajes ( $5.5 \%$ cada una) fueron la utilidad del conocimiento que brinda la carrera, el interés por defender a otros, la posibilidad de ayudar a otros y el sentido de justicia. 

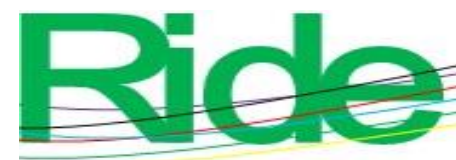

Revista Iberoamericana para la Investigación y el Desarrollo Educativo

ISSN 2007 - 7467

Al cuestionar a los graduados sobre su interés por estudiar un posgrado, $53.3 \%$ de los de semiescolarizado respondieron que sí tenían esa intención, mientras que $6.6 \%$ ya contaba con uno. Entre los egresados del sistema escolarizado, $88.8 \%$ manifestaron un interés por hacerlo. Tomando en cuenta las edades de los egresados, así como el hecho de que aquellos del sistema semiescolarizado ya cuentan con una carrera anterior o decidieron esta profesión debido a la modalidad de estudios, se puede comprender que el interés por un posgrado sea menor, aunque sigue siendo un porcentaje que representa a más de la mitad de este grupo. La inversión en el capital humano es dispar entre ambos grupos, lo que indica que el objetivo por el que ingresaron a esta carrera es también muy distinto.

Uno de los puntos más interesantes de este estudio es el que atañe al trabajo y escolaridad de los padres, pues se hallaron diferencias interesantes entre ambos casos. Por un lado, para los graduados de semiescolarizado, $26.6 \%$ de los padres y $33.3 \%$ de las madres contaban con primaria trunca, aunque $13.3 \%$ de los padres y $13.3 \%$ de las madres sí finalizaron sus estudios de primaria. De los padres, $6.6 \%$ contaba con secundaria, y este mismo porcentaje se repitió en el caso de preparatoria, licenciatura y maestría. Entre las madres, $6.6 \%$ tenía la preparatoria y $13.3 \%$ la licenciatura (es necesario señalar que no todos respondieron a las preguntas sobre la formación y trabajo de sus padres).

Figura 2. Motivos de elección de carrera

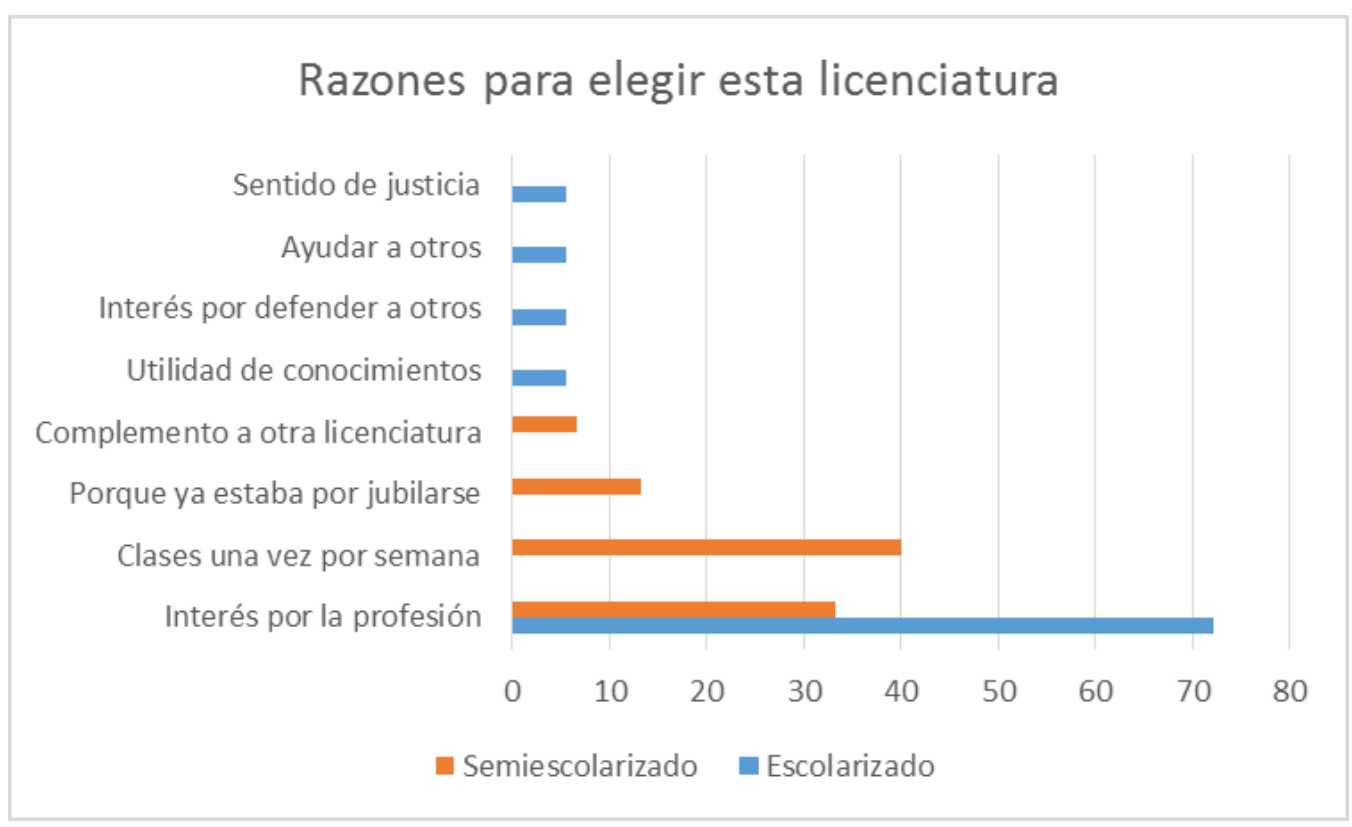

Fuente: Elaboración propia 

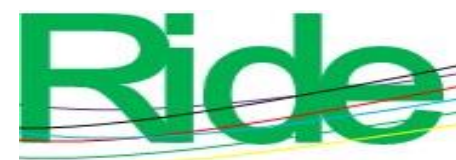

Revista Iberoamericana para la Investigación y el Desarrollo Educativo

ISSN 2007 - 7467

Entre los padres de los egresados de escolarizado, $50 \%$ de los padres contaba con su primaria completa, mientras que $44.4 \%$ de las madres también tenía ese grado académico. Ninguno de los padres tenía primaria trunca y solo $5.55 \%$ de las madres no había culminado esa etapa educativa. Asimismo, $11.1 \%$ de los padres tenía estudios de secundaria, $5.55 \%$ de preparatoria, $5.55 \%$ de carrera técnica, $16.6 \%$ de licenciatura y $5.55 \%$ de maestría. Entre las madres, $16.6 \%$ tenían estudios de secundaria, $11.1 \%$ de preparatoria, $5.55 \%$ de licenciatura trunca y $16.6 \%$ de licenciatura finalizada. Llama la atención que el nivel de escolaridad es ligeramente mayor en el caso de los padres de aquellos estudiantes de modalidad escolarizada.

Cabe señalar que existen diferencias de edad entre los egresados; en tal sentido, podríamos asumir que existen diferencias entre las edades de sus padres y que, por lo tanto, existe una diferencia entre el nivel educativo porque en las últimas décadas se ha impulsado por lograr un nivel más alto (Jiménez y Pacheco, 2018).

Figura 3. Nivel de escolaridad de los padres

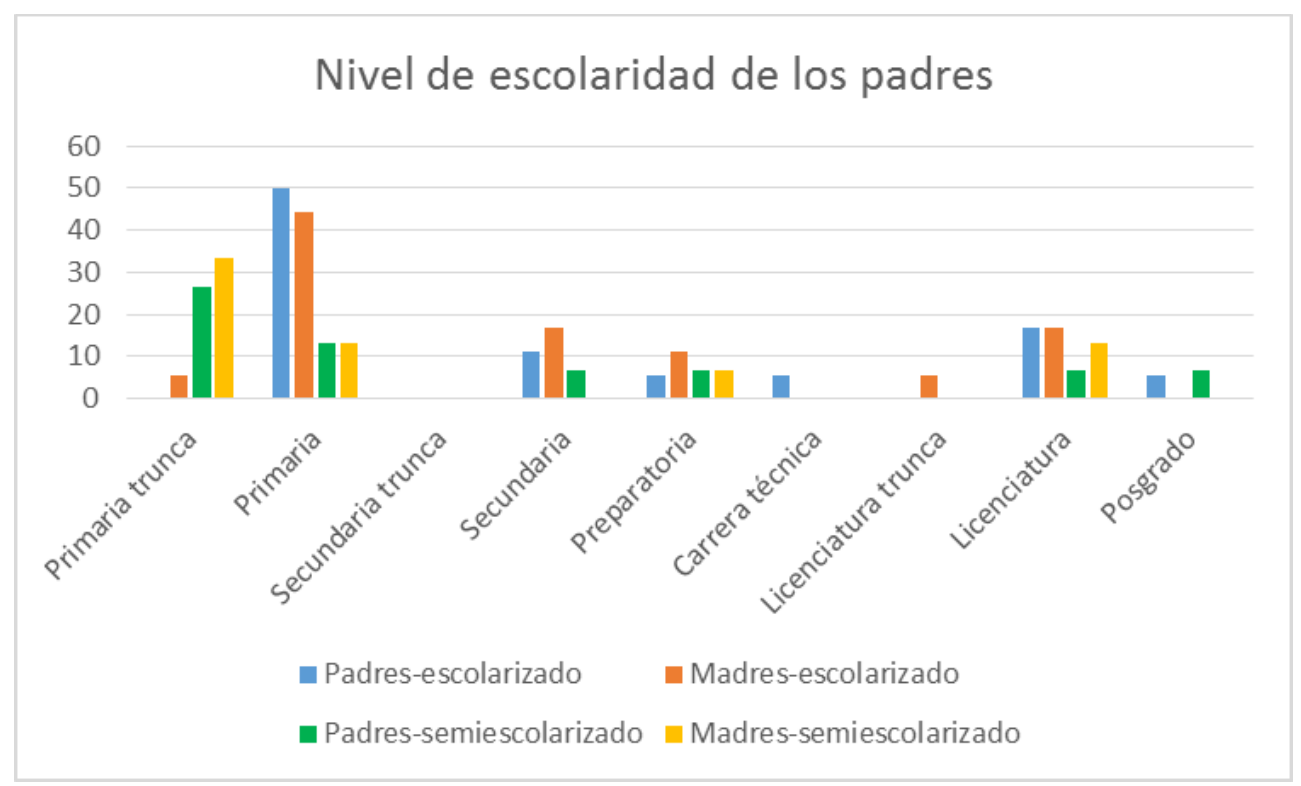

Fuente: Elaboración propia

En la figura 4 se aprecian los siguientes resultados: en el caso de los egresados del sistema escolarizado, $16.6 \%$ representa a los padres que son obreros, porcentaje que empata con aquellos que trabajan en el campo (como agricultor o ganadero); los que se dedican al comercio y los que son empleados (no se especificó en qué área o sector). El 11.11 \% son 

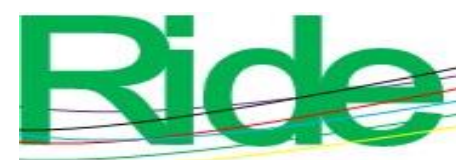

Revista lberoamericana para la Investigación y el Desarrollo Educativo

ISSN 2007 - 7467

jubilados y también compartiendo porcentajes se encuentran los maestros, empleada doméstica, servidor público, veterinarios y abogados, con 11.1 \% cada uno. Llama la atención que un porcentaje tan bajo corresponda a la profesión elegida por los hijos.

En cuanto a los egresados de la modalidad semiescolarizada, $6.6 \%$ son maestros, con el mismo porcentaje se encuentran los comerciantes, empleados y abogados (nuevamente, un porcentaje muy pequeño con respecto a la totalidad), mientras que $20 \%$ de los padres están jubilados y $33.3 \%$ se dedican a trabajar en el campo.

Figura 4. Ocupación de los padres

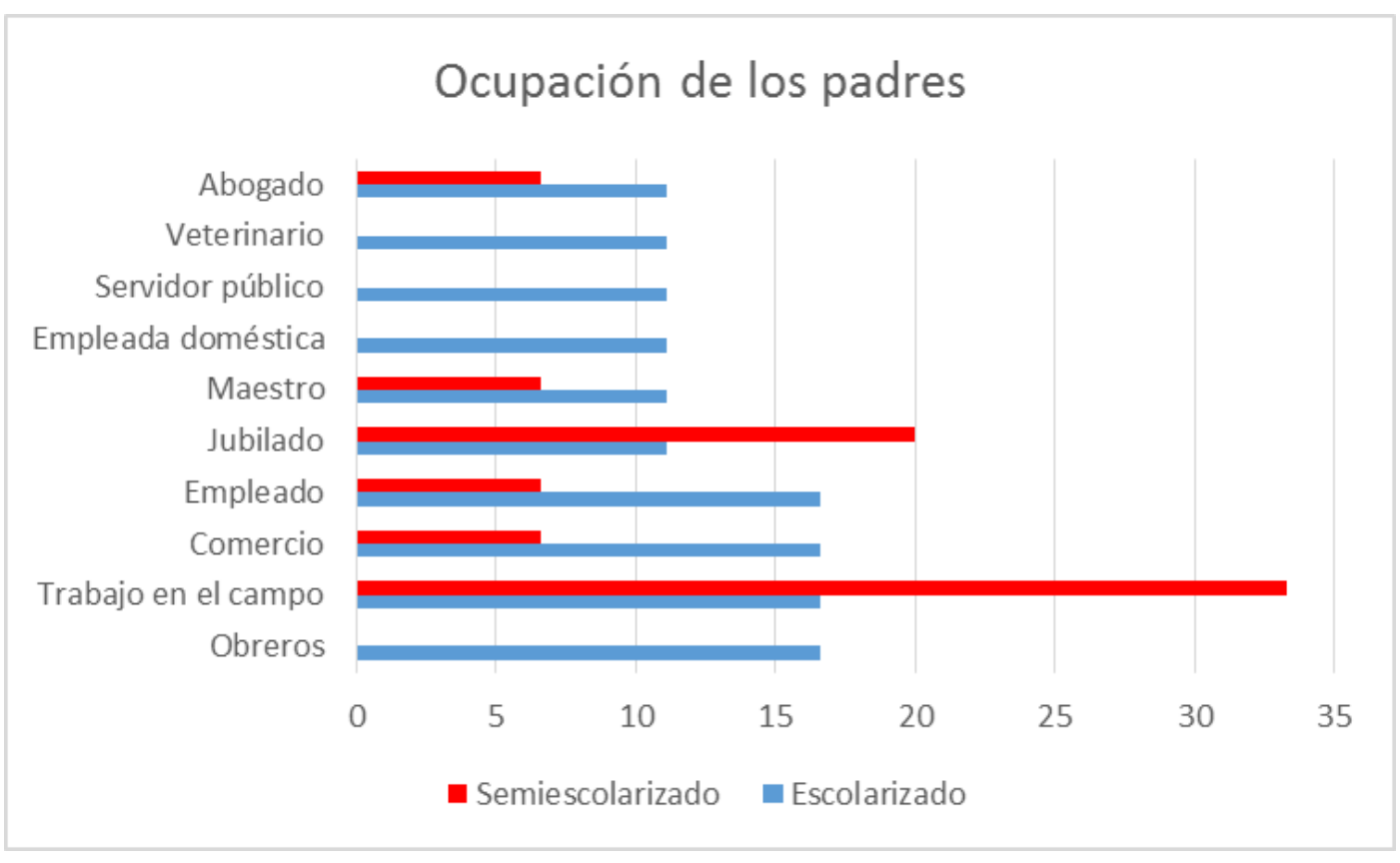

Fuente: Elaboración propia

Una de las grandes diferencias entre los encuestados se encontró en la manera en la que solventaron sus estudios. Es uno de los puntos en los que se hacen manifiestas las diferencias entre los perfiles personales que tenían los egresados cuando eran estudiantes. Entre los que asistían a clases de lunes a sábado, $16.6 \%$ se sostuvo gracias al apoyo de sus padres, $27.7 \%$ logró hacerlo con la ayuda de sus padres y su trabajo, $22.2 \%$ con la ayuda de padres y el apoyo de una beca, $16.6 \%$ solo con una beca, mientras que $5.55 \%$ lo hizo por sí mismo. Los trabajos desempeñados a la par de sus estudios fueron los siguientes: empleados en comercios, asistentes en despachos jurídicos y trabajos en negocios familiares. 

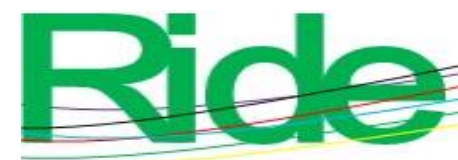

Revista Iberoamericana para la Investigación y el Desarrollo Educativo

ISSN 2007 - 7467

En cuanto a los de semiescolarizado, 66.6 \% se solventó a sí mismo los gastos, $13.3 \%$ recibió ayuda de sus padres, $13.3 \%$ lo hizo por medio del apoyo de una beca y su propio trabajo, y $6.6 \%$ gracias a que su cónyuge pagó sus estudios. Entre los que trabajaban, se desempeñaban en distintos sectores laborales, muchos de ellos en los relacionados a su primera licenciatura.

Figura 5. Formas de manutención durante sus estudios

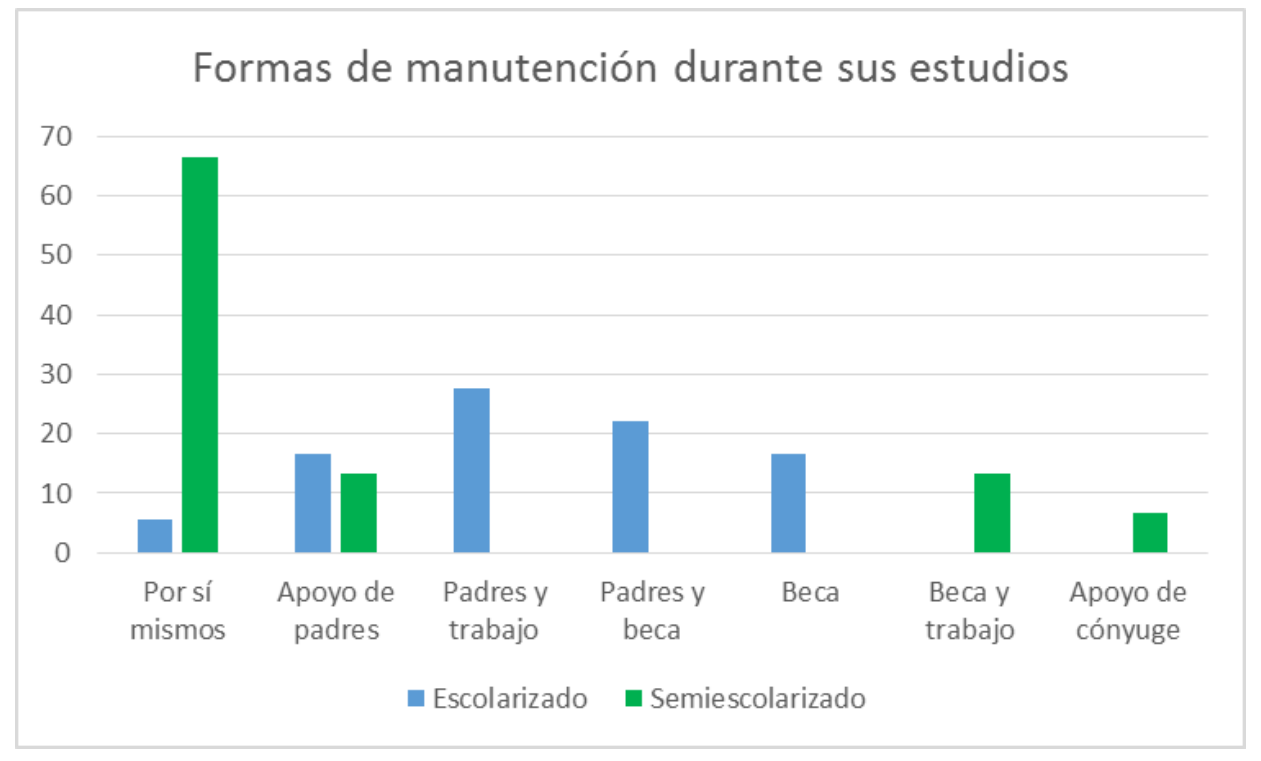

Fuente: Elaboración propia

En los resultados es posible encontrar que la adquisión de una licenciatura sí fue redituable en el caso de ambos grupos, es decir, la inversión en su propio capital sí se tradujo como una mejora en sus condiciones laborales. Es curioso destacar que mientras los del grupo escolarizado explican cuántos trabajos han tenido, los del semiescolarizado se limitan a indicar que ya trabajaban con anterioridad (66.6\% de los casos). En la tabla 1 aparece el porcentaje que corresponde al número de trabajos que han tenido los egresados; se toman en cuenta aquellos trabajos que tenían desde antes de estudiar una licenciatura (en el caso de los egresados de semiescolarizado, el porcentaje total no corresponde al $100 \%$ porque no respondieron sobre el número de trabajos que han tenido). 
Tabla 1. Número de trabajos

\begin{tabular}{|c|c|c|}
\hline $\begin{array}{c}\text { Número de } \\
\text { trabajos }\end{array}$ & Escolarizado & Semiescolarizado \\
\hline 1 & $0 \%$ & $20 \%$ \\
\hline 2 & $22.2 \%$ & $6.6 \%$ \\
\hline 3 & $27.7 \%$ & $0 \%$ \\
\hline 4 & $16.6 \%$ & $6.6 \%$ \\
\hline 5 & $16.6 \%$ & $6.6 \%$ \\
\hline 6 o más & $5.5 \%$ & $0 \%$ \\
\hline
\end{tabular}

Fuente: Elaboración propia

Uno de los puntos de mayor interés fue conocer cómo los egresados obtuvieron su empleo. Para el caso de los graduados de la carrera de Abogado en sistema escolarizado, $72.2 \%$ lo obtuvo gracias a la recomendación que alguien más hizo de ellos, $5.5 \%$ cumplía con los requisitos y el resto no respondió a esta pregunta. En el caso de semiescolarizado, $13.3 \%$ lo hizo por recomendación de alguien más y solo 6.6 \% cumplía con los requisitos (el resto no respondió a esta pregunta).

En la figura 6 se observa cómo el título ayudó a que los egresados se posicionaran dentro del mercado laboral: $46.6 \%$ de los de semiescolarizado declararon que el título sí les ayudó para hacerlo, 26.6 \% declaró que no, y el resto (26.8 \%) no respondió al respecto. Para los egresados de escolarizado, $55.5 \%$ dijo que el título le ayudó a conseguir trabajo, mientras que $11.1 \%$ dijo que no; el resto $(33.3 \%)$ no respondió. 

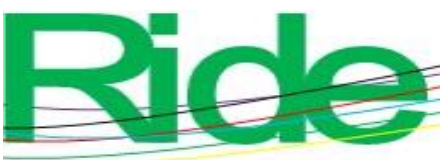

Revista Iberoamericana para la Investigación y el Desarrollo Educativo ISSN 2007 - 7467

Figura 6. Ayuda del título para emplearse

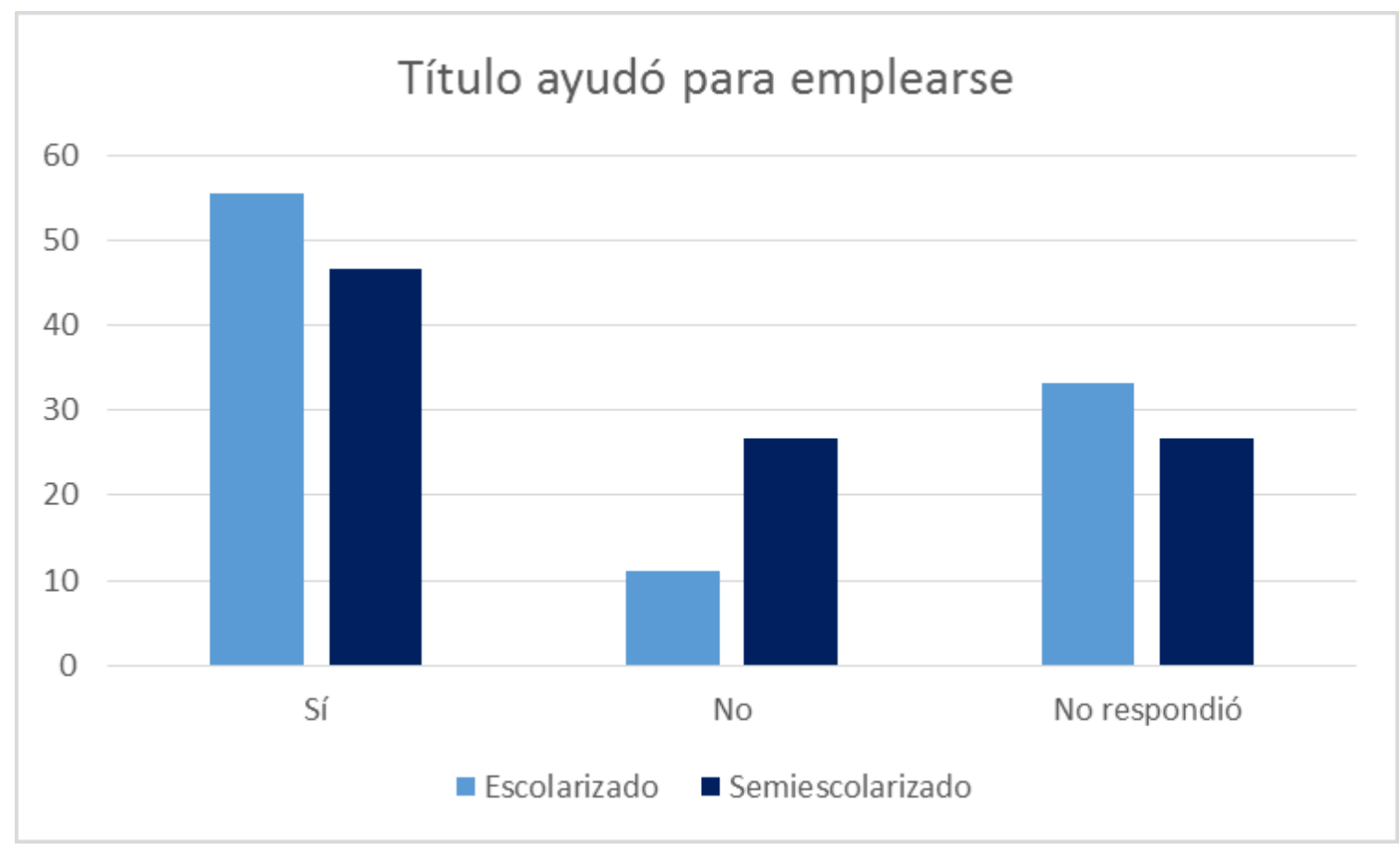

Fuente: Elaboración propia

Resulta significativo que la proporción es cercana a la mitad en ambos casos, es decir, la mitad de los egresados encontró empleo por contar con un título universitario. Sobre si el título se relacionaba con su carrera, solo una tercera parte $(33.3 \%)$ de egresados de semiescolarizado dijeron que sí, aunque habrá de tomarse en cuenta que algunos ya contaban con una profesión anterior o con un empleo que no se relacionaba necesariamente con el derecho. Además, entre estos egresados, solo $66.6 \%$ manifestó estar laborando, por lo tanto, encontramos que la mitad de ellos trabajan en un área relacionada con su profesión.

Para el caso de los egresados del sistema escolarizado, $66.6 \%$ dijo que sí hallaba una relación entre su trabajo y la profesión que estudiaron (el doble que los de la otra modalidad de estudios). De la totalidad de los entrevistados, $77.7 \%$ se encuentra laborando, así que la relación entre trabajo y profesión se vuelve mucho mayor (solo $11.1 \%$ trabajarían en un área no vinculada con el derecho). 
Figura 7. Ascenso en el trabajo

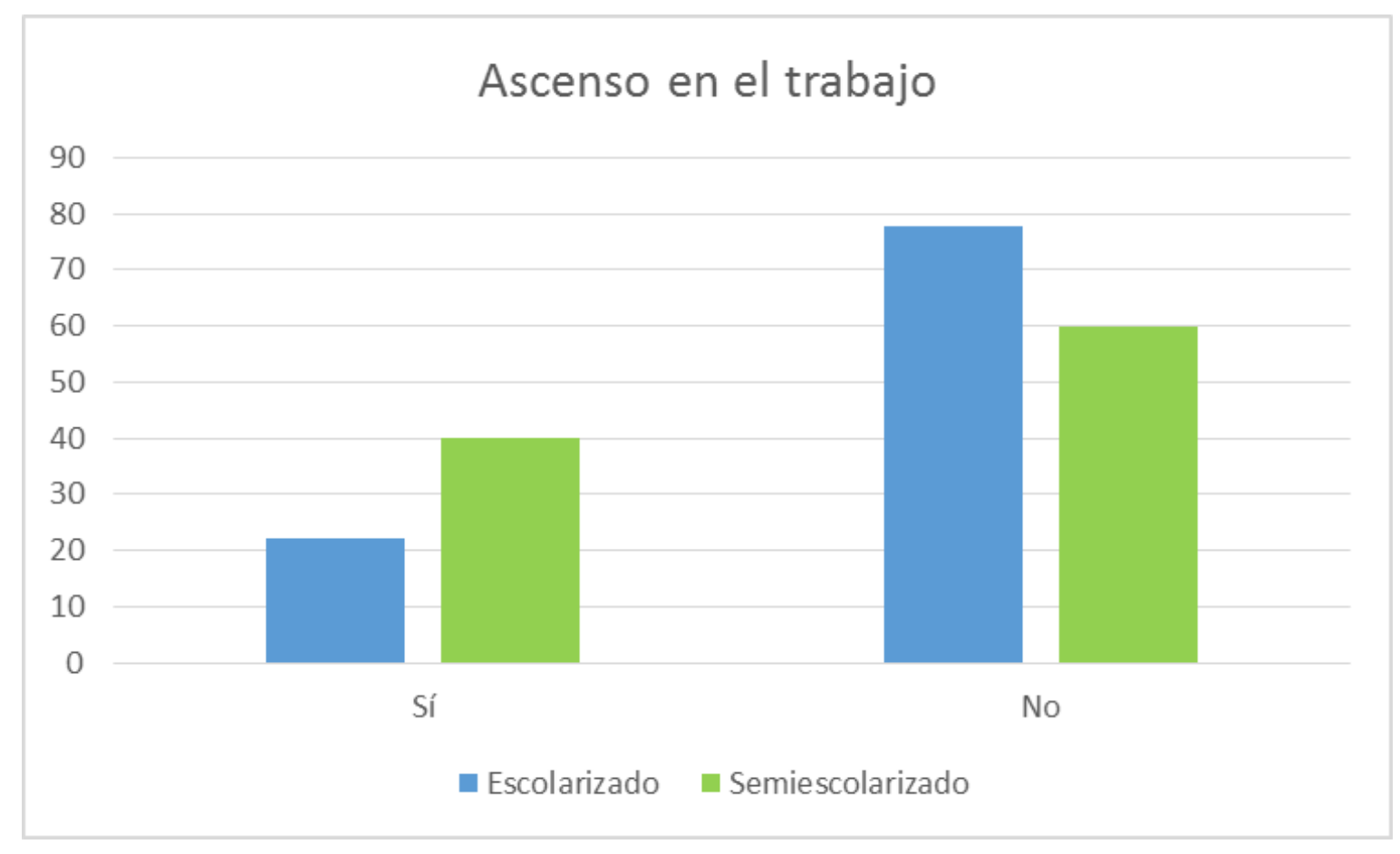

Fuente: Elaboración propia

Vistos los porcentajes anteriores, se puede decir que el panorama es satisfactorio, aun cuando la mayor proporción de los egresados obtuvieron su empleo gracias a una recomendación o por sus prácticas o servicio social (en este caso, la universidad relaciona de manera más profunda a sus estudiantes con la sociedad). De hecho, en la figura 7 se señaló el porcentaje de egresados que han ascendido y los que no lo han hecho dentro de sus trabajos. Curiosamente, $40 \%$ de graduados de semiescolarizado lograron ascender, mientras que solo $22.2 \%$ de escolarizado lo hicieron, a pesar de que estos últimos fueron quienes tuvieron acceso a una educación más continua.

Finalmente, se les pidió a los egresados sus opiniones con respecto a las materias cursadas a lo largo de sus estudios universitarios, así como que explicaran cuáles eran, desde sus puntos de vista, los mayores problemas que tuvieron durante su formación a nivel superior. Para facilitar la lectura de los resultados, se presentan las tablas 2 y 3. 


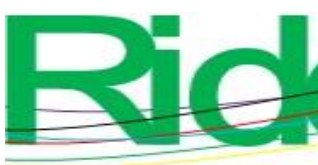

Revista lberoamericana para la Investigación y el Desarrollo Educativo ISSN 2007 - 7467

Tabla 2. Materias que deberían desaparecer del plan de estudio

\begin{tabular}{|l|c|c|}
\hline \multicolumn{1}{|c|}{$\begin{array}{c}\text { Materias que deberían } \\
\text { desaparecer }\end{array}$} & $\begin{array}{c}\text { Egresados del sistema } \\
\text { escolarizado }\end{array}$ & $\begin{array}{c}\text { Egresados del sistema } \\
\text { semiescolarizado }\end{array}$ \\
\hline Derecho administrativo & $8.33 \%$ & $0 \%$ \\
\hline Derecho ambiental y agrario & $33.3 \%$ & $0 \%$ \\
\hline Derecho bancario & $8.33 \%$ & $0 \%$ \\
\hline Derecho familiar & $0 \%$ & $20 \%$ \\
\hline Derecho mercantil & $8.33 \%$ & $0 \%$ \\
\hline Derecho procesal & $8.33 \%$ & $0 \%$ \\
\hline Filosofía & $0 \%$ & $60 \%$ \\
\hline Seminario de investigación & $25 \%$ & $20 \%$ \\
\hline Teoría del Estado & $8.33 \%$ & $0 \%$ \\
\hline TOTAL & $\mathbf{1 0 0 \%}$ & $\mathbf{1 0 0} \%$ \\
\hline
\end{tabular}

Fuente: Elaboración propia

Las respuestas de ambos grupos varían. Aunque existe una coincidencia en la sugerencia de eliminar la materia Seminario de Investigación, el porcentaje no es el mayor en ambos grupos. Mientras que para el grupo semiescolarizado la materia Filosofía debería desaparecer del plan de estudios, para el grupo escolarizado es la de Derecho Ambiental y Agrario la que debería hacerlo. La concentración de respuestas del grupo semiescolarizado en tres materias podría indicar también una mala experiencia con los profesores o con la manera en la que estas fueron trabajadas en la modalidad que los estudiantes atendieron. Por otro lado, en el caso de los que asistieron a clases de forma escolarizada, hubo una diversidad de respuestas.

En cuanto a las materias que, a su parecer, son imprescindibles en el plan de estudios, en la tabla 3 encontramos los porcentajes correspondientes. Las respuestas de ambos grupos nuevamente presentan coincidencias, como al elegir las materias Derecho Penal y Derecho Civil entre las más favorecidas. Las materias Derecho Procesal, Derecho Familiar y Amparo siguen en porcentajes. Las diferencias que podemos encontrar en ambos grupos están por un lado en el favorecimiento que el escolarizado hace de las materias Derecho Mercantil, Introducción al Derecho, Teoría General del Derecho y Derecho Romano, las cuales no fueron mencionadas por el grupo semiescolarizado. De forma inversa, para estos últimos las materias Delitos, Ciencia Penitenciaria, Derecho Fiscal, Inglés y Redacción y Argumentación Jurídica fueron mencionadas, pero los de escolarizado no las consideraron

\footnotetext{
${ }^{2}$ Esta pregunta no fue respondida por todos los egresados; sólo $66.6 \%$ del sistema escolarizado y $26.6 \%$ del semiescolarizado dieron una respuesta. Se consideran las respuestas como totales para obtener los porcentajes.
} 
entre sus respuestas. En síntesis, se puede señalar que fueron más las materias señaladas como importantes dentro del plan de estudios de la carrera, por lo que se sugiere que no sean eliminadas.

Tabla 3. Materias imprescindibles durante su formación

\begin{tabular}{|l|c|c|}
\hline \multicolumn{1}{|c|}{ Materias imprescindibles } & $\begin{array}{c}\text { Egresados del sistema } \\
\text { escolarizado }\end{array}$ & $\begin{array}{c}\text { Egresados del } \\
\text { sistema } \\
\text { semiescolarizado }\end{array}$ \\
\hline Amparo & $9.09 \%$ & $9.52 \%$ \\
\hline Ciencia penitenciaria & $0 \%$ & $4.76 \%$ \\
\hline Delitos & $0 \%$ & $4.76 \%$ \\
\hline Derecho administrativo & $2.27 \%$ & $0 \%$ \\
\hline Derecho civil & $18.18 \%$ & $23.8 \%$ \\
\hline Derecho constitucional & $4.54 \%$ & $0 \%$ \\
\hline Derecho familiar & $6.81 \%$ & $14.28 \%$ \\
\hline Derecho fiscal & $0 \%$ & $4.76 \%$ \\
\hline Derecho laboral & $4.54 \%$ & $4.76 \%$ \\
\hline Derecho mercantil & $6.81 \%$ & $0 \%$ \\
\hline Derecho penal & $22.72 \%$ & $14.28 \%$ \\
\hline Derecho procesal & $4.54 \%$ & $9.52 \%$ \\
\hline Derecho romano & $4.54 \%$ & $0 \%$ \\
\hline Inglés & $0 \%$ & $4.76 \%$ \\
\hline Introducción al derecho & $6.81 \%$ & $0 \%$ \\
\hline Redacción y argumentación jurídica & $0 \%$ & $4.76 \%$ \\
\hline Teoría general del derecho & $6.81 \%$ & $0 \%$ \\
\hline TOTAL & $\mathbf{1 0 0 \%}$ & $\mathbf{1 0 0 \%}$ \\
\hline
\end{tabular}

Fuente: Elaboración propia

Finalmente, y para ahondar en las comparaciones entre ambos sistemas educativos, se preguntó a los egresados acerca de los problemas que tuvieron a lo largo de su formación. Los obstáculos percibidos variaron, y se presentan en la tabla 4. 


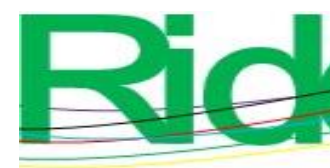

Revista Iberoamericana para la Investigación y el Desarrollo Educativo ISSN 2007 - 7467

Tabla 4. Problemas en la formación

\begin{tabular}{|c|c|c|}
\hline Problemas en la formación & $\begin{array}{l}\text { Egresados del sistema } \\
\text { escolarizado }\end{array}$ & $\begin{array}{c}\text { Egresados del } \\
\text { sistema } \\
\text { semiescolarizado }\end{array}$ \\
\hline Ausentismo de profesores & $16.6 \%$ & $20 \%$ \\
\hline Capacitación de profesores & $44.4 \%$ & $13.3 \%$ \\
\hline Falta de laboratorios de idiomas & $0 \%$ & $13.3 \%$ \\
\hline Falta de sala de juicios orales & $22.2 \%$ & $0 \%$ \\
\hline $\begin{array}{l}\text { Falta de tiempo para realizar servicio } \\
\text { social y prácticas }\end{array}$ & $0 \%$ & $26.6 \%$ \\
\hline Lejanía del centro universitario & $5.55 \%$ & $6.6 \%$ \\
\hline Préstamo de libros por poco tiempo & $0 \%$ & $6.6 \%$ \\
\hline $\begin{array}{c}\text { Problemas con la unidad de } \\
\text { asignación de servicio social }\end{array}$ & $11.1 \%$ & $0 \%$ \\
\hline Cancelación del acto académico & $0 \%$ & $6.6 \%$ \\
\hline $\begin{array}{l}\text { Maestros que desalientan a los } \\
\text { alumnos }\end{array}$ & $0 \%$ & $6.6 \%$ \\
\hline TOTAL & $100 \%$ & $100 \%$ \\
\hline
\end{tabular}

Fuente: Elaboración propia

Las respuestas a la pregunta sobre los problemas a lo largo de su formación pueden ser explicadas por la forma en que se desarrollaron las clases en las dos modalidades. Por un lado, los egresados del sistema escolarizado se quejaron en su mayoría por la falta de capacitación de maestros, mientras que el problema mayor para los de semiescolarizado fue el ausentismo de los profesores (esta situación se presentó también con el otro grupo, aunque no fue considerada tan problemática como la falta de capacitación).

\section{Discusión}

Se hallaron diferencias en distintas características y circunstancias en las que los egresados llevaron a cabo sus estudios. Por ejemplo, en la manera de costearlos la disparidad entre los resultados hace manifiesto el rol que tienen los padres para que los más jóvenes puedan seguir con sus estudios, mientras que aquellos que atendieron clases solo una vez por semana trabajaban en su gran mayoría. Puede ser que gracias a su trabajo pudieron llevar a cabo su formación, aunque vale acotar que este sistema no se ofrece en la mayoría de las licenciaturas.

Sobre el rol de los padres, también se encontró relación entre la formación de los padres y el éxito laboral y escolar de los hijos, lo cual ya ha sido analizado en otros trabajos (De Vries y Navarro, 2011). Sin embargo, en los dos casos que fueron revisados en esta 


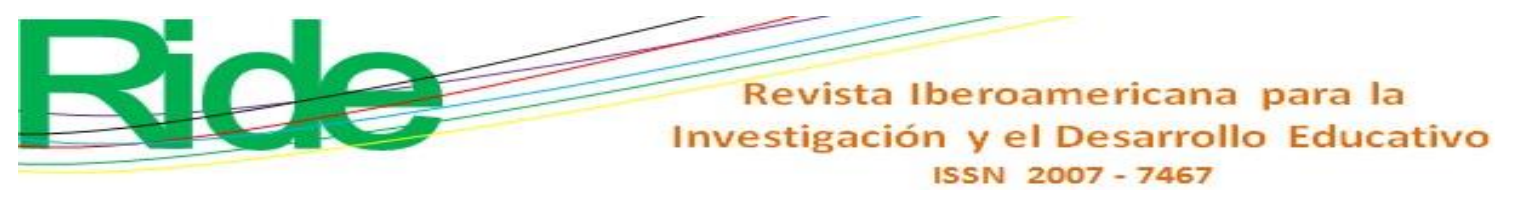

indagación, se puede apreciar que los egresados son hijos de obreros, personas que trabajan en el campo o que se emplean en diferentes oficios (en menor porcentaje son profesionistas). Además, cabe destacar que pocos progenitores se dedican al oficio de la abogacía, por lo que no podrían ayudar de manera directa a los hijos para que se desenvuelvan en el mundo laboral.

Asimismo, en el caso del grupo semiescolarizado se puede decir que la adquisición de un título universitario sirvió para conseguir un nivel más alto que el de los progenitores. En el caso del escolarizado, aunque con menor medida hubo una diferencia entre padres e hijos con respecto al tipo de trabajo que desempeñan, se puede afirmar que los egresados en ambos grupos eligieron una carrera que no se relacionaba directamente con las de sus padres. Este punto es de interés, ya que podemos entrever que son los estudiantes los que se deciden por una profesión a partir de cuestiones que no necesariamente están relacionadas con el trabajo de los padres.

Con base en la teoría del capital humano, se esperaría que los egresados continuaran con su formación a través de cursos, congresos y prácticas en espacios destinados a la abogacía. Los resultados que esperarían estos egresados deberían ser redituables con respecto a la inversión que han hecho (Ontiveros, 2007).

Si bien hay coincidencias entre la experiencia de ambos grupos — por ejemplo, al explicar que hubo deficiencias en su formación por la falta de laboratorios-, existen discrepancias en cuanto a sus experiencias particulares. Por un lado, los de escolarizado explicaron la necesidad de contar con una sala de juicios orales, mientras que los de semiescolarizado lo hicieron sobre la falta de un laboratorio de idiomas. En el momento en el que ambos grupos se encontraban estudiando, la sala de juicios orales estaba en construcción, mientras que el laboratorio de idiomas ya existía en el campus, pero no ofrecía sus servicios los sábados, por lo que los egresados de semiescolarizado no pudieron acceder a él.

El desarrollo de sus prácticas y servicio social fue difícil para ambos grupos, siendo el semiescolarizado el que tuvo problemas para llevarlo a cabo debido a sus propios tiempos (muchos de ellos trabajaban en un horario que les impedía realizarlo), mientras que para el escolarizado los problemas tuvieron que ver con la unidad de servicio social que les tenía que asignar las plazas o los lugares donde habrían de llevarlos a cabo. Finalmente, algunos de los problemas que fueron exclusivos para los egresados de semiescolarizado tuvieron que ver 


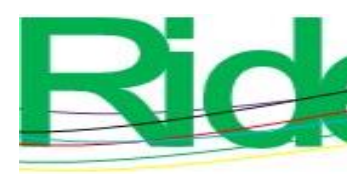

Revista Iberoamericana para la Investigación y el Desarrollo Educativo

ISSN 2007 - 7467

con los horarios de clases y las dificultades para acceder a servicios del Cualtos (como el préstamo de libros o el uso del laboratorio de idiomas).

Más importante aún fue el hecho de que algunos de los egresados manifestaron que les fue cancelado el acto académico un día antes de su realización, además de que hubo profesores que frecuentemente les desalentaban a seguir estudiando en un proyecto semiescolarizado. Algunas de estas problemáticas fueron mencionadas por aquellos egresados que fueron contactados y se negaron a participar. Ellos argumentaron el poco apoyo recibido por parte de la universidad, y se puede visualizar que las problemáticas que tuvieron como estudiantes podrían haber sido solucionadas si se les hubieran ofrecido servicios adecuados a sus horarios y modalidad de estudios.

Finalmente, vale retomar los datos de De Vries y Navarro (2011), quienes señalan que el índice de desempleo de los egresados universitarios es de $11 \%$. Aunque el porcentaje es menor al que encontramos entre los graduados de la carrera de Abogado en Cualtos, es posible que se deba a la negativa a responder a determinadas preguntas en las entrevistas, especialmente por parte de los de semiescolarizado. Entre los argumentos mencionados, el más recurrente se refería al trato descortés que recibieron por parte del personal del campus, de ahí que no se sintieran con la disposición para colaborar en el trabajo.

Por último, es importante destacar que en diversos estudios se ha mostrado que si bien la abogacía es una carrera con altos niveles de ocupación, emplearse durante el primer año de haber egresado ha tenido un impacto más favorable en su trayectoria. Así como aquellos que se especializaron en un área particular, ya sea con la práctica dentro de su formación como estudiantes de licenciatura, o ya con un posgrado (Chamorro, Molina, Burbano y Cadena, 2019; Mendoza, González y Heredia 2020; Observatorio Laboral, 2015). Aunque las intenciones de los egresados que pertenecían a ambos grupos al momento de ingresar y estudiar la carrera podían diferir, sin duda las experiencias que vivieron durante su tránsito por la licenciatura no fueron del todo favorables para uno de los grupos. Independientemente de las razones por las que estudiaban, las circunstancias en las que lo hicieron se diferenciaron, llevando con ello a que la calidad de sus estudios universitarios fuera dispar. 


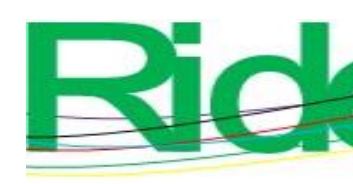

Revista Iberoamericana para la Investigación y el Desarrollo Educativo ISSN $2007-7467$

\section{Conclusiones}

El análisis de dos grupos que estudiaron la carrera de Abogado en un mismo periodo y campus (en este caso, el Cualtos), aunque en diferentes modalidades (escolarizada y semiescolarizada), ha servido para comprender cómo esta última variable puede influir en la formación y percepción de los alumnos. Si bien la integración al mundo laboral no parece estar condicionada de forma directa por la modalidad de formación (ya que a ambos grupos el título de licenciatura les ayudó para emplearse), es posible entrever que la elección de esta carrera fue determinada por las posibilidades que una u otra opción ofrecían. En otras palabras, los alumnos no suelen seleccionar esta profesión por la influencia de los padres, ya que la mayoría no se dedican a ello.

De hecho, el origen social tampoco fue determinante para ingresar a la vida laboral, ya que ninguno de los egresados cuyos padres eran abogados expresó que hubieran usado esa influencia para conseguir un trabajo. Sin embargo, el apoyo de los padres para que los egresados pudieran estudiar sí fue determinante en muchos casos, especialmente para aquellos que estudiaron la carrera de Abogado de forma escolarizada.

En cuanto a las materias que deberían ser eliminadas del plan de estudios, se hallaron opiniones dispares en ambos grupos. Al respecto, cabe señalar que el currículo de la carrera de Abogado no fue el mismo para los egresados entrevistados en este trabajo.

Por otra parte, los problemas que tuvieron los egresados cuando eran estudiantes constituyen un fuerte indicador de cómo la modalidad de estudios elegida puede influir en la manera en la que se desarrolla la carrera. Por ejemplo, los estudiantes de semiescolarizado dijeron que habían sido desanimados por los profesores. Esto provocó una negativa al momento de intentar entrevistarlos para desarrollar este trabajo. La falta de motivación o de empatía por parte de quienes los acompañaron en este trayecto fue uno de los temas recurrentes.

A pesar de esto, sobresale que los egresados se abrieron paso en el ámbito universitario y laboral gracias a sus propios esfuerzos. La búsqueda de becas, el sustento por sí mismos, etc., demuestra el interés por llevar a cabo sus estudios de nivel superior. Asimismo, y aunque una gran mayoría de los egresados obtuvieron su empleo por recomendación (en especial los escolarizados), vale subrayar que esta no es una realidad exclusiva de los abogados o de los profesionistas de la región. De hecho, se puede inferir que 

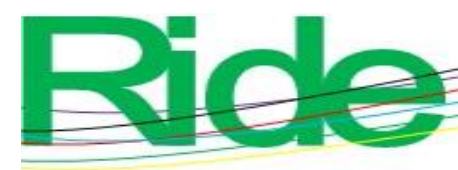

Revista Iberoamericana para la Investigación y el Desarrollo Educativo

ISSN $2007-7467$

esas recomendaciones provinieron de profesores, compañeros de trabajo o amigos, ya que sus padres no laboraban en ese campo.

En definitiva, ambos grupos tienen coincidencias en su formación, en las maneras en las que obtuvieron sus empleos y sobre todo en su opinión sobre su propia carrera (materias importantes, imprescindibles, lugares de trabajo, razones de elección de su profesión). Sin embargo, la modalidad de estudios tuvo influencias importantes para ambos grupos, por lo que la disparidad se profundizó y las diferencias se hicieron patentes, aun cuando tuvieron en muchos casos a los mismos profesores, programas de materias y recursos. En pocas palabras, estudiar una licenciatura en un mismo campus universitario se puede convertir en una experiencia totalmente distinta si se realiza en diferentes modalidades escolares.

\section{Referencias}

Chamorro, D., Molina, T., Burbano, L. y Cadena, A. (2019). Seguimiento a graduados: posibilidades de empleo, competencias y funciones de los abogados (UNIANDES Ibarra). Debate Jurídico Ecuador. Revista Digital de Ciencias Jurídicas de UNIANDES, 2(2), 149-166.

De Vries, W. y Navarro, Y. (2011). ¿Profesionistas del futuro o futuros taxistas? Los egresados universitarios y el mercado laboral en México. Revista Iberoamericana de Educación Superior, 2(4).

González, C. y Villanueva, G. (2007). Seguimiento de egresados para la Licenciatura en Informática del Centro Universitario de los Altos. Universidad de Guadalajara.

González, C. y Ortega, G. (2015). Empleabilidad de los egresados de la Carrera de Contaduría Pública. RIDE. Revista Iberoamericana para la Investigación y el Desarrollo Educativo, 6(11).

Guerrero, M. (2020). Teoría y realidad del capital humano en México: alcances y consecuencias de la educación por competencias. Revista Internacional de Estudios sobre Sistemas Educativos, 2(100), 465-486.

Instituto Nacional de Estadística y Geografía (Inegi) (2015). Sistema Nacional de Clasificación de Ocupaciones (SINCO) (2011). Recuperado de http://www.inegi.org.mx/est/contenidos/proyectos/aspectosmetodologicos/clasificad oresycatalogos/sinco.aspx 


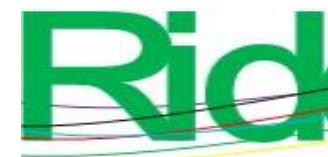

Revista Iberoamericana para la Investigación y el Desarrollo Educativo

ISSN 2007 - 7467

Jaramillo, A., Pineda, A. G. y Ortiz Correa, J. S. (2006). Estudios sobre egresados. La experiencia de la Universidad EAFIT. Revista Universidad EAFIT, 42(141), 111-124.

Jiménez, C. y Pacheco, J. (2018). Resultados de la educación en México desde el enfoque del capital humano: impactos a nivel local en los estados con proceso electoral para gobernador en 2018. RIEL, 2(3) 59-76.

Mendoza, L., González, K. y Heredia, R. (2020). Las prácticas profesionales y las competencias del egresado: análisis del programa educativo de Derecho Internacional en la UAPCI-UAEMÉX. Revista de Investigación Latinoamericana en Competitividad Organizacinal, 2(5).

Observatorio Laboral (2015). Perfil ocupacional del abogado. Recuperado de http://www.observatoriolaboral.gob.mx/static/estudios-publicaciones/ Perfil_abogado.html

Organización para la Cooperación y Desarrollo Económicos (OCDE) (2010). Habilidades y competencias del siglo XXI para los aprendices del nuevo milenio en los países de la $O C D E$. España: Instituto de tecnologías educativas.

Ontiveros, M. (2007). El mercado laboral como vínculo entre la inversión en educación y su rendimiento. Revista Latinoamericana de Estudios Educativos, 37(3-4), 239-268.

Schultz, T. (1983). La inversión en capital humano. Educación y Sociedad, 8(3).

Székely, M. y Flores, I. (2018). Educación y desarrollo en México: una historia de baja capacidad de aprovechamiento del capital humano. Washington, DC: IDB Working Paper Series. Inter-American Development Bank (IDB).

Villalobos, G. y Pedroza, R. (2009). Perspectiva de la teoría del capital humano acerca de la relación entre educación y desarrollo económico. Tiempo de Educar, 10(20) 273-306. 